\title{
Public repository with Monte Carlo simulations for high-energy particle collision experiments
}

\section{Sergei Chekanov*}

HEP Division, Argonne National Laboratory, 9700 S. Cass Avenue, Argonne, IL 60439, USA

E-mail: chekanoveanl.gov

\begin{abstract}
Planning high-energy collision experiments for the next few decades requires extensive Monte Carlo simulations in order to accomplish physics goals of these experiments. Such simulations are essential for understanding fundamental physics processes, as well as for setting up the detector parameters that help establish R\&D projects required over the next few decades. This paper describes a public repository with Monte Carlo event samples before and after detector-response simulation. The goal of this repository is to facilitate the accomplishment of many goals in planning a next generation of particle experiments.
\end{abstract}

38th International Conference on High Energy Physics 3-10 August 2016

Chicago, USA

\footnotetext{
* Speaker.
} 


\section{Introduction}

The High-Luminosity Large Hadron Collider (HL-LHC) can answer many essential questions of high-energy physics (HEP) over the next few decades. Other possible HEP projects are the High-Energy LHC (HE-LHC), Large Hadron electron Collider (LHeC), International Linear Collider (ILC), Circular Electron Positron Collider (CEPC) in China, Super Proton-Proton Collider (SPPC), and the Future Circular Collider at CERN (FCC-ee, FCC-ep, and FCC-hh). Although the technical plans of these projects should still be shaped, the fact that they are on the map of possible projects shows that HEP is a vibrant field with promising future. To secure this future, exploratory physics studies and R\&D projects focused on detector designs should be established. Such studies require Monte Carlo collision events after fast or full (Geant4) simulation of detector response. Analysis of these Monte Carlo simulations after a realistic event reconstruction is crucial for understanding physics processes, exploring technology choices, detector parameters, and for seeding new hardware R\&D projects.

Due to the diversity of future HEP experiments, leveraging Monte Carlo simulations created by a large community of researchers is an effective way to advance physics studies, detector designs and analysis techniques. The HepSim project [1] described in this paper is a recent attempt to create an open-access data catalogue with Monte Carlo predictions for the HEP community. This paper describes some of its features.

\section{HepSim repository}

The goal of the HepSim repository is to provide the HEP community with events from Monte Carlo event generators, as well as with events after full and fast detector simulations. This data repository was started at ANL during the US long-term planning study of the American Physical Society's Division of Particles and Fields (Snowmass 2013). Currently, HepSim hosts about 220 Monte Carlo event samples, totaling 1.6 million events. A single data sample contains a set of files from Monte Carlo event generators with fully documented information on Monte Carlo settings. The data created by event generators will further be called EVGEN events. A fraction of EVGEN events have been processed through fast and full detector simulations using several detector designs.

HepSim repository uses the computational capabilities of the Chicago area, such as Openscience grid Connect (OSG-CI, U.Chicago/ANL), BlueGene/Q supercomputer of the Argonne Leadership Computing Facility, Argonne's Laboratory Computing Resource Center (LCRC), and ATLAS support center (HEP/ANL). Several data storages are provided by the HEP/ANL, Univ. of Chicago (OSG-CI) and the Science Gateways at NERSC.

The user front-end of the HepSim database is shown in Figure 1. The web interface of HepSim allows searching for the needed processes and datasets. The files can be downloaded using the "hstools" Java package as described in [2]. In addition to the user-friendly set of tools for searching and downloading files, the package can be used for a quick processing of EVGEN events in order to create the needed kinematic distributions and cross sections for physics studies. For example, the tool includes histogram classes, plot canvases for $2 \mathrm{D} / 3 \mathrm{D}$ plots, and particle classes and jet algorithms [2]. Data analysis can be performed using the Jas4pp program [3], which originates 


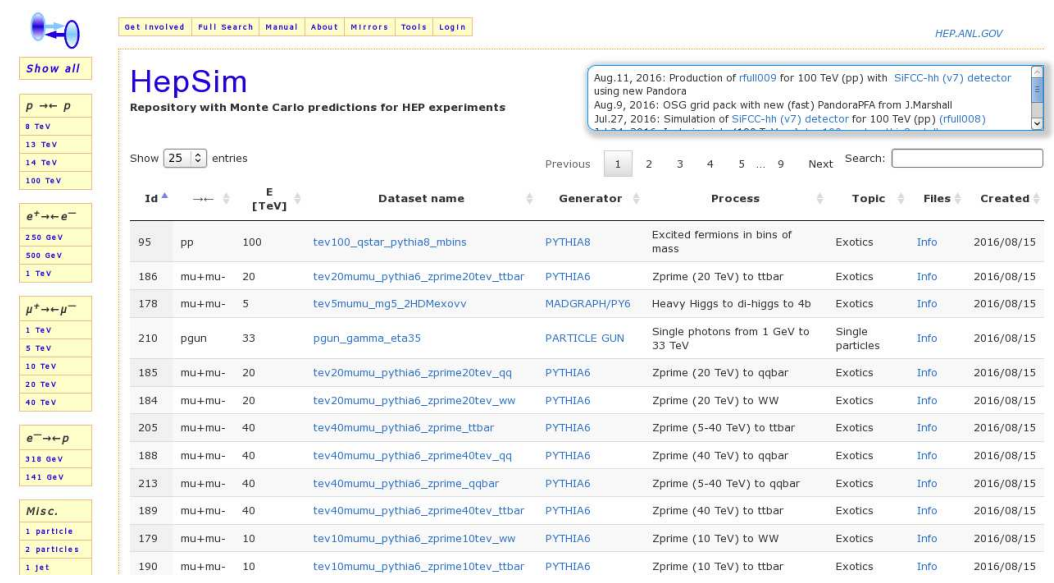

Figure 1: Illustration of the front-end of HepSim where a user can select the needed process.

from the Jas3 program developed at SLAC for the ILC. This program supports data analysis of EVGEN events, as well as analysis of the data after full simulations to be described later. This package also provides the Wired 4 event display. The common Java API shared by the Jas4pp and "hs-tools" is described in [4]. To perform a complete data analysis, the needed C++ packages are also available from the ProMC package [5].

\subsection{EVGEN events}

Created EVGEN events are kept in the archive file format called ProMC [5]. This binary format was designed to keep unweighted events from parton-shower event generators and weighted events from next-to-leading order QCD calculations. In addition, the original log files created during the generation step are included. To keep file sizes small, a variable-length integer encoding scheme ("varints") is used.

Open access to ProMC files with EVGEN events is the central part of the HepSim design, since further fast and full event simulations are performed using computer resources from multiple locations. The compact files designed for web streaming, together with the http protocol optimized for handling many (relatively small) files, is one of the distinct features of HepSim compared to other production systems.

\subsection{Full Geant4 simulations}

A number of detector descriptions have been created for HepSim using the "silicon detector" (SiD) detector designed for the ILC project. This detector combines excellent silicon tracking with high-granular electromagnetic and hadronic calorimeters which are required for identification of separate particles.

A family of "all-silicon" detectors was created using the "compact.xml" geometry files in the XML format. Such geometry files can be converted to the Geometry Description Markup Language (GDML) format for easy viewing and debugging using ROOT. Figure 2 shows the cross sections of four detectors available from the HepSim GDML repository. Some of these detectors, such as SiFCC (a "performance" detector for a $100 \mathrm{TeV}$ collider), have several production versions. 

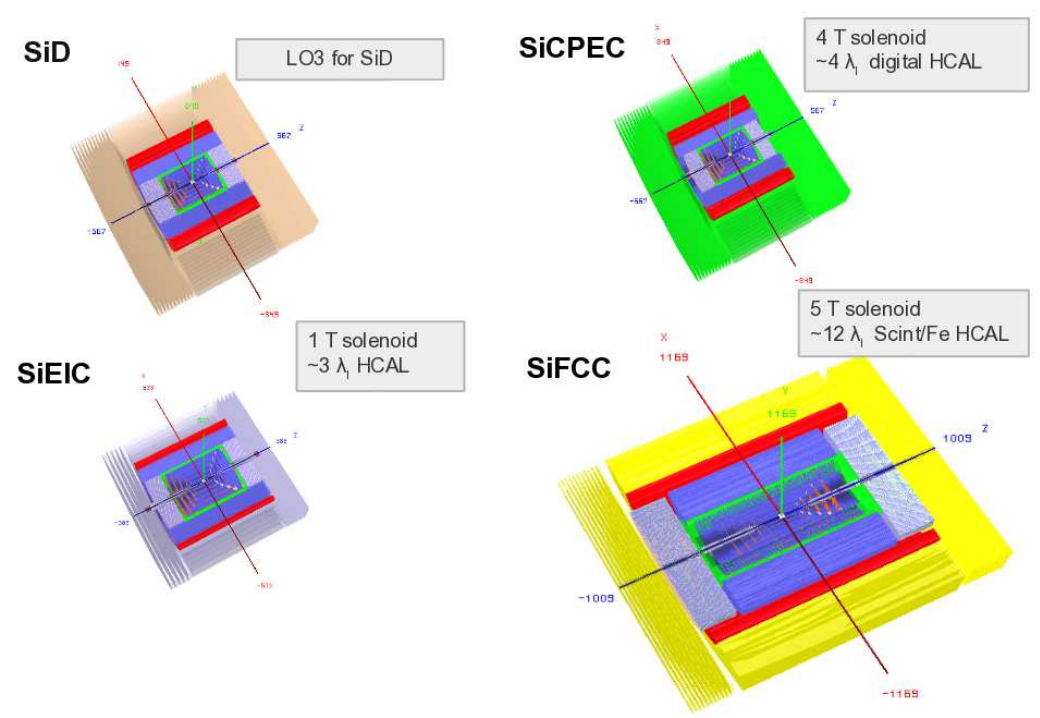

Figure 2: This graphic shows cross-sections of the four detectors available from HepSim. They are all designed using the "all-silicon" concept inspired by the SiD detector. The red color shows the solenoid which encloses hadronic, electromagnetic and outer and inner tracker. The muon detector is located behind the solenoid. This image retains the relative sizes of the shown detectors.

The response of the detectors to physics processes is simulated using the Simulator for the Linear Collider (SLIC) package [6] developed for the ILC project. The main strength of this software lies in the fact that detector geometries can easily be changed using the XML files. The final output of the reconstruction step is the particle-flow objects created by the Pandora Particle Flow algorithm [7, 8]. In addition, complete information on reconstructed tracks, calorimeter clusters and calorimeter hits is preserved for data analysis. The reconstructed events are kept in the original SLIC format, called LCIO [12].

Currently, less than $0.01 \%$ of EVGEN events hosted by HepSim were simulated and reconstructed using HepSim detector designs. These simulations are typically required to verify detector performances and detector response to physics processes. The processing step ProMC $\rightarrow$ LCIO that creates events with fully reconstructed tracks, calorimeter clusters and particle flow objects can take up to $4 \mathrm{CPU} *$ hours per event for $100 \mathrm{TeV} p p$ collisions (without pileup) in the SiFCC detector which features the high-granularity calorimeters and silicon trackers. A memory usage of up to $16 \mathrm{~GB}$ is required. We do not expect a significant increase of the number of simulated and reconstructed events in the near future due to the lack of general-purpose computing resources.

Data analysis of fully simulated and reconstructed events can be done with the platformindependent Jas4pp program using the Java or Python programming languages. There is also a dedicated $\mathrm{C}++$ package to be compiled on the Linux platform.

\subsection{Fast simulations}

The fast detector simulation uses the DELPHES framework [9] that incorporates a tracking system, magnetic field, calorimeters, and a muon system. DELPHES simulates the calorimeter 
system by summing together cells to form towers ${ }^{1}$. Currently, three types of detectors are supported: a CMS-like detector, an ATLAS-like detector, and the detector developed during the US long-term planning study of the future program of particle physics (Snowmass 2013) [10]. The output from the fast simulation is kept in the ROOT files. About $1 \%$ of the EVGEN files were processed with the DELPHES program. The fast simulation is more than a factor $10^{5}$ faster compared to the full SLIC simulation described above, but DELPHES simulation often lacks important information needed for understanding impact of detector performance on physics processes.

\section{Conclusions}

To increase the pace of scientific discoveries in high-energy particle physics and to foster designs of new HEP experiments, HepSim datasets can freely be downloaded for physics and detector-performance publications without any restrictions. The HepSim Monte Carlo data repository has been readily accessible to the public since 2014. In addition to the data files, the HepSim online manual contains code snippets, which can easily be incorporated into data-analysis projects. Recently, there were several studies based on HepSim data: CEPC studies [11] using the "all-silicon" concept based on the SiD detector are exclusively based on HepSim samples. Singleparticle samples for the ATLAS tracking upgrade for the HL-LHC experiment were created using the HepSim tools. Several studies based on full detector simulations included in HepSim were presented during this conference $[13,14,15]$. The repository was also used to initiate the creation of Monte Carlo samples for several physics papers by the ATLAS collaboration. Currently, HepSim is an indispensable tool for FCC-hh physics and detector performance studies since the unprecedented energy regime imposes new requirements on detector technologies which can be studied using detailed Geant4 simulations.

\section{Acknowledgments}

The author would like to thank many people who provided software codes and Monte Carlo samples for inclusion into the HepSim repository. Their names are listed on the HepSim web page. Argonne National Laboratory's work was supported by the U.S. Department of Energy, Office of Science under contract DE-AC02-06CH11357.

\section{References}

[1] S. Chekanov, HepSim: a repository with predictions for high-energy physics experiments, Advances in High Energy Physics, 2015 (2015) 136093, available as http: //atlaswww.hep.anl.gov/hepsim/.

[2] S. V. Chekanov, I. Pogrebnyak, D. Wilbern, Cross-platform validation and analysis environment for particle physics, (2016) (submitted to Comp. Phys. Comm.), arXiv:1510. 06638.

[3] Jas4pp. Java Analysis Studio for Particle Physics, https: / / atlaswww.hep.anl.gov/asc/jas 4pp/ (2016).

\footnotetext{
${ }^{1}$ Towers are regions defined in pseudorapidity $\eta$ and azimuthal angle $\phi$.
} 
[4] S. V. Chekanov, Numeric Computation and Statistical Data Analysis on the Java Platform, Springer, London, 2016, ISBN 978-3-319-28531-3.

[5] S. V. Chekanov, E. May, K. Strand, P. Van Gemmeren, ProMC: Input-output data format for HEP applications using varint encoding, Comp. Phys. Commun. 185 (2013) 2629.

[6] N. Graf and J. McCormick, Simulator for the linear collider (SLIC): A tool for ILC detector simulations, AIP Conf. Proc. 867 (2006) 503.

[7] M. J. Charles, PFA Performance for SiD, in: Linear colliders. Proceedings, International Linear Collider Workshop, LCWS08, and International Linear Collider Meeting, ILC08, Chicago, USA, Novermber 16-20, 2008, 2009. arXiv:0901.4670.

[8] J. S. Marshall and M. A. Thomson, Pandora Particle Flow Algorithm, in: Proceedings, International Conference on Calorimetry for the High Energy Frontier (CHEF 2013), 2013, pp. 305.

[9] J. de Favereau, et al., DELPHES 3, A modular framework for fast simulation of a generic collider experiment, JHEP 1402 (2014) 057.

[10] J. Anderson, et al., Snow mass Energy Frontier Simulations, in: Proceedings, Community Summer Study 2013: Snowmass on the Mississippi (CSS2013), Minneapolis, MN, USA, July 29-August 6, 2013, arXiv:1309.1057.

[11] S. V. Chekanov and M. Demarteau, Conceptual Design Studies for a CEPC Detector, arXiv: 1604 .01994. (2016). A white paper contributed to the IAS Program on High Energy Physics (4-29 Jan, 2016).

[12] F. Gaede et al., LCIO: A persistency framework for linear collider simulation studies, Talk from the 2003 Computing in High Energy and Nuclear Physics (CHEP03), La Jolla, Ca, USA, March 2003, arXiv:physics/0306114.

[13] Y. Shin-Shan, et al., Study Of Boosted W-Jets And Higgs-Jets With the SiFCC, A talk given at the ICHEP16 conference.

[14] S. Sen, et al., Tau reconstruction for a FCC experiment, ICHEP16 poster.

[15] N. V. Tran, et al., High-granularity calorimeter for a FCC detector, ICHEP16 poster. 